\title{
Valutazione economica dello studio CARDS: un aggiornamento
}

Simona de Portu (1), Sabato Montella ${ }^{(1)}$, Paolo Cortesi ${ }^{(2)}$, Enrica Menditto ${ }^{(1)}$, Lorenzo G. Mantovani (1)

\begin{abstract}
Introduction: in the last decades, prevalence and incidence of type II diabetes mellitus have been rapidly growing worldwide. Most recent projections estimate that the number of people affected by diabetes is destined to double in 2030, producing a significant increase of the healthcare expenditure for the management of complications. Prevention of cardiovascular events in diabetes population represents a priority for decision makers, who have to evaluate the cost-effectiveness of therapeutic interventions.

Objective: to provide an updated cost-effectiveness evaluation of treating type II diabetes patients with atorvastatin versus placebo, in the light of the imminent price reduction of atorvastatin due to loss of exclusivity and of other therapeutic and hospital costs.

Material and Methods: we derived clinical information from the CARDS study, a randomized, multicenter clinical trial evaluating efficacy of atorvastatin versus placebo in preventing the occurrence of cardiovascular events in a cohort of type II diabetes patients without previous history of coronary events. A cost-effectiveness analysis in the perspective of the National Healthcare System (SSN) has been performed, under the hypothesis of the imminent price reduction of atorvastatin, due to the loss of exclusivity.

Results: after a median follow up of 3.9 years, the number of patients with at least a major cardiovascular event requiring hospitalization was lower in the atorvastatin arm (5.8\%) compared to the placebo arm $(9.0 \%$; $\mathrm{p}=0.001$ ). Based on a cohort of 1,000 patients, treatment with atorvastatin permitted to gain 29.28 life years. The incremental cost of adding atorvastatin to the standard therapy amounted to $€ 305,682$, and was partially balanced by a cost reduction due to fewer hospitalizations, compared to the placebo arm ( $€ 168,313)$. Total direct costs were of $€ 602.186$ in the atorvastatin group and of $€ 464,818$ in the placebo group, resulting into an incremental cost-effectiveness ratio of $€$ 4,692 for Life Year Gained (LYG).

Conclusion: the present study is an update of a previous economic analysis of the CARDS trial. Under the assumed new cost scenario, the cost-effectiveness profile of treating diabetic patients with atorvastatin becomes highly favourable, and leads to a significant reduction of the cost for Life Year Gained compared to the previous findings.
\end{abstract}

Keywords: cost-effectiveness, atorvastatin, type II diabetes, CARDS trial Farmeconomia e percorsi terapeutici 2011; 12(Suppl 2): 35-40

\section{INTRODUZIONE}

Il diabete è una delle cause principali di morbidità e mortalità nella maggior parte dei paesi del mondo. Il numero di soggetti affetti da diabete è costantemente in aumento: la crescita demografica, il progressivo invecchiamento della popolazione, l'urbanizzazione e i conseguenti effetti sulla prevalenza di obesità e inattività fisica costituiscono le principali cause del crescente numero di casi [1]. Nella proiezione epidemiologica proposta da Wild e colleghi nel 2005 [2], si è stimato che la prevalenza complessiva del diabete è destinata a passare dal $2,8 \%$ nel 2000 al 4,4\% nel 2030. In termini assoluti, il numero di soggetti affetti da diabete dovrebbe crescere dai 171 milioni dell'anno 2000 ai 366 milioni dell'anno 2030. Altri modelli di valutazione della mortalità [1] hanno stimato che, nel 2010, il numero di decessi attribuibili al diabete sarà di circa 4 milioni nei soggetti con età compresa tra i 20 e i 79 anni. L'impatto della mortalità del diabete è simile a quello attribuibile alle malattie infettive. Nel $50 \%$ dei casi, la morte di un soggetto diabetico è causata da malattia aterosclerotica. Da uno studio condotto in Italia alla fine degli anni ' 90 [3] risulta che la prevalenza del diabete diagnosticato in Italia è del $3 \%$ circa mentre l'incidenza del diabete mellito è di 2,2 per 1.000 persone per anno $(2,0$ per gli uomini e 2,4 per le donne).

La conoscenza dell'impatto epidemiologico del diabete e dei costi associati alla gestione delle complicanze è di fondamentale importanza per un'appropriata allocazione delle risorse eco-

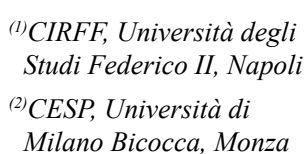

Milano Bicocca, Monza 
nomiche in sanità. Uno studio di costo di malattia pubblicato nel 2002 [4] e condotto in otto paesi europei (Belgio, Francia, Germania, Italia, Paesi Bassi, Spagna, Svezia e Regno Unito) ha stimato che i costi totali diretti per la gestione del diabete mellito di tipo II ammontano a 29 miliardi di euro. Il costo annuale per la gestione di un paziente è pari a $€ 2.384$ e il $55 \%$ dei costi è attribuibile a ricoveri ospedalieri, che hanno riguardato il $13 \%$ del totale dei soggetti. Una recente revisione delle valutazioni economiche condotte in Italia sul diabete [5] ha evidenziato che il costo annuale di un paziente affetto da diabete è compreso tra i 2.300 e i 3.000 euro. La spesa per farmaci impatta per circa il $22-34 \%$ dei costi totali, mentre il $52-60 \%$ è attribuibile a ricoveri ospedalieri che, nella maggior parte dei casi, sono causati da eventi cardiovascolari, i quali inducono un aumento dei costi del $70-100 \%$. La riduzione del colesterolo LDL e l'aumento del colesterolo HDL sono considerati obiettivi prioritari per la prevenzione degli eventi cardiovascolari nei soggetti diabetici. Le linee guida della European Society of Cardio$\operatorname{logy}[6]$ e della Società Italiana di Diabetologia [7] raccomandano l'utilizzo degli inibitori delle HMG-CoA reduttasi (statine), indipendentemente dal livello di LDL nei pazienti in prevenzione secondaria, e in considerazione del rischio cardiovascolare globale nei soggetti in prevenzione primaria. Il trattamento farmacologico con atorvastatina ha dimostrato di prevenire significativamente l'insorgenza di eventi ischemici in soggetti diabetici in prevenzione sia primaria sia secondaria $[8,9]$.

Il presente lavoro costituisce un aggiornamento di una precedente analisi [10] che ha valutato l'impatto economico dell'impiego di atorvastatina nello studio CARDS. Le analisi valutano i costi e i benefici complessivi del trattamento con atorvastatina alla luce della futura riduzione di prezzo di cessione di atorvastatina al SSN, dovuta a scadenza brevettuale (prevista per la fine del 2011) e delle variazioni dei costi per i ricoveri ospedalieri avvenute rispetto al tempo della precedente analisi.

\section{MATERIALI E METODI}

La valutazione economica è stata realizzata ricavando le informazioni cliniche dallo studio CARDS. La misurazione dei costi è stata effettuata utilizzando le informazioni economiche sui costi delle terapie e dei ricoveri attualmente praticati in Italia.

\section{Dati clinici}

I dati clinici necessari per la valutazione sono ricavati dallo studio CARDS [9], che ha confrontato l'efficacia di atorvastatina rispetto a placebo nel prevenire l'occorrenza di eventi ischemici. Lo studio CARDS è un trial clinico, multicentrico, randomizzato, in doppio cieco, che ha arruolato soggetti con diabete di tipo II, non affetti da malattia cardiovascolare conclamata, di età compresa tra i 40 e i 75 anni. Per essere inclusi nello studio, i soggetti dovevano registrare una concentrazione di colesterolo LDL minore o uguale a $4,14 \mathrm{mmol} / \mathrm{l}$, livelli di trigliceridi non superiori $6,78 \mathrm{mmol} / \mathrm{l}$ e presentare almeno una tra le seguenti condizioni: retinopatia, albuminuria, anamnesi positiva per il fumo, ipertensione. I soggetti inclusi nello studio sono stati assegnati al trattamento standard più atorvastatina $10 \mathrm{mg} / \mathrm{die}(\mathrm{n}=1.410) \mathrm{o}$, in alternativa, al trattamento standard più placebo $(\mathrm{n}=1.428)$. Il $9 \%$ dei soggetti assegnati al gruppo placebo ha comunque ricevuto un trattamento ipolipemizzante in aperto, prima della fine dello studio. L'endpoint primario è stato il tempo all'insorgenza di uno dei seguenti eventi: sindrome coronarica acuta, rivascolarizzazione, ictus.

\section{Dati di costo}

Nell'analisi sono stati considerati i costi diretti sanitari derivanti dall'impiego della terapia farmacologica e dalla gestione degli eventi acuti che hanno richiesto un ricovero ospedaliero. I costi unitari utilizzati nell'analisi sono riportati in Tabella I. Il costo per la terapia farmacologica con atorvastatina è stato calcolato quantificandone l'utilizzo secondo lo schema posologico indicato nello studio CARDS, tenendo comun-

\begin{tabular}{llc}
\hline \multicolumn{1}{c}{ Variabili } & Fonte & Costi unitari (€) \\
\hline Infarto miocardico fatale e non & Mantovani, 2009 [12] & $6.022,00$ \\
Angina instabile & DRG 140 & $2.121,22$ \\
Morte per altra causa CV & DRG 129 & $3.841,81$ \\
Ictus fatale e non fatale & DRG 14 & $3.926,62$ \\
Rivascolarizzazione coronarica (PTCA/CABG) & DRG 106,107, 517, 518* & $6.829,34$ \\
Atorvastatina 10 mg/die & Riduzione del 50\% rispetto all'attuale prezzo di cessione SSN [11] & 0,35 \\
\hline
\end{tabular}

\section{Tabella I}

Elenco dei costi unitari usati nella valutazione economica

$\mathrm{CABG}=$ bypass aortocoronarico; $\mathrm{DRG}=$ Diagnosis Related Group; PTCA = angioplastica coronarica

* media ponderata per la frequenza di ospedalizzazioni come risulta dalle schede di dimissione, anno 2007 
que in considerazione che una certa percentuale di soggetti inizialmente arruolati nel gruppo atorvastatina $(15 \%)$, non ha mai ricevuto il farmaco in studio. Il costo giornaliero di atorvastatina $10 \mathrm{mg}$ è stato calcolato considerando una riduzione del $50 \%$ dell'attuale prezzo di cessione al SSN [11], ossia la riduzione di prezzo attesa a seguito della scadenza del periodo di copertura brevettuale. Il costo del ricovero per infarto miocardico acuto è stato desunto da un recente studio condotto su banche dati amministrative della Regione Lombardia [12]. In assenza di stime simili per le altre tipologie di evento, il costo delle restanti ospedalizzazioni è stato valutato calcolando la media dei DRG regionali specifici per l'evento, ponderata per la frequenza dei ricoveri nelle singole regioni. Dai dati dello studio non è stato possibile distinguere le frequenze di infarto del miocardio e di angina instabile nei due gruppi di trattamento. Per procedere alla misurazione dei costi, il numero complessivo degli eventi nei due gruppi di trattamento è stato moltiplicato per un costo unitario medio per evento coronarico acuto (Tabella I). Non è stato possibile, con le informazioni a disposizione, calcolare $\mathrm{i}$ costi relativi alla gestione degli eventi avversi. I costi generati dopo sono espressi in Euro 2010.

\section{Tecnica di valutazione}

La tecnica di valutazione economica utilizzata nel presente lavoro è l'analisi costoefficacia $[13,14]$, che confronta le conseguenze economiche e cliniche del trattamento con atorvastatina, rispetto alle alternative di confronto di ciascuno degli studi clinici. L'indicatore utilizzato nell'analisi costo-efficacia è
l'ICER (Incremental Cost-Effectiveness Ratio), che esprime il costo necessario per evitare un evento con atorvastatina rispetto all'alternativa in studio, ed è calcolato sulla base del rapporto tra la differenza dei costi e la differenza di efficacia clinica tra le due alternative in studio. La prospettiva utilizzata nell'analisi è quella del Servizio Sanitario Nazionale (SSN). L'orizzonte temporale utilizzato per la valutazione economica coincide con il periodo di osservazione degli studi clinici analizzati. I costi e gli effetti clinici sono stati calcolati su ipotetiche coorti di 1.000 soggetti per gruppo di trattamento. La robustezza dell' analisi è stata valutata attraverso un'analisi di sensibilità del $\pm 10 \%$ su costi per la terapia farmacologica e i ricoveri ospedalieri $[15,16]$. Una seconda analisi di sensibilità è stata effettuata modificando il tasso di sconto annuale al $5 \%$ e $0 \%$ rispetto all'analisi principale (sconto annuale del 3\%). È stata condotta, infine, un'analisi di soglia, allo scopo di calcolare il costo giornaliero di atorvastatina per cui i due trattamenti in studio generino gli stessi costi sanitari.

\section{RISULTATI}

Lo studio CARDS si è concluso 2 anni prima del previsto per il raggiungimento precoce della differenza di efficacia predeterminata tra i due gruppi di trattamento. Il follow up mediano è stato pari a 3,9 anni. Il trattamento con atorvastatina $10 \mathrm{mg} / \mathrm{die}$ ha ridotto del $37 \%$ il rischio di occorrenza di almeno un evento cardiovascolare rispetto a placebo (IC95\% $=-52 \%,-17 \%$; $\mathrm{p}=0,001)$. Complessivamente, 58 soggetti su 1.000 randomizzati ad atorvastatina e 90 sog-

\begin{tabular}{|c|c|c|c|c|c|}
\hline \multirow{2}{*}{ Variabili } & \multicolumn{2}{|c|}{ Placebo* } & \multicolumn{2}{|c|}{ Atorvastatina 10 mg* } & \multirow{2}{*}{ Differenza* $(€)$} \\
\hline & n. & Costi (€) & n. & Costi (€) & \\
\hline Infarto miocardico (fatale e non) & 43,26 & $249.362,69$ & 23,11 & $133.200,69$ & $-116.162,00$ \\
\hline Morte per altra causa CV & 2,84 & $10.431,74$ & 7,00 & $25.750,62$ & $15.318,88$ \\
\hline Angina instabile & 6,38 & $12.959,54$ & 4,90 & $9.952,59$ & $-3.006,95$ \\
\hline Rivascolarizzazione coronarica (PTCA/CABG) & 12,77 & $83.447,29$ & 8,40 & $54.930,29$ & $-28.517,00$ \\
\hline Ictus (fatale e non) & 24,82 & $88.197,56$ & 14,71 & $52.251,49$ & $-35.946,07$ \\
\hline Costi totali per ospedalizzazioni & & $444.398,82$ & & $276.085,68$ & $-168.313,14$ \\
\hline Costo trattamento & & $20.418,76$ & & $326.100,37$ & $305.681,61$ \\
\hline Costi totali & & $468.817,58$ & & $602.186,05$ & $137.368,47$ \\
\hline Pazienti con almeno un evento CV & 90,07 & - & 58,12 & - & - \\
\hline Anni di vita guadagnati (LYG) & - & - & 29,28 & - & - \\
\hline Costo per paziente libero da malattia (€/pz) & 4.299 & & & & \\
\hline Costo per LYG (€/LYG) & 4.692 & & & & \\
\hline
\end{tabular}

\section{Tabella II}

Studio CARDS: analisi costo-efficacia (i costi sostenuti negli anni successivi al primo sono scontati del 3\%)

* dati riferiti a un'ipotetica coorte di 1.000 soggetti 
getti su 1.000 randomizzati a placebo hanno sperimentato almeno un evento clinico (infarto del miocardio, rivascolarizzazione, angina instabile, ictus).

I costi sostenuti per la terapia e la gestione degli eventi clinici sono riportati in Tabella II. Il costo totale per la terapia farmacologica è stato pari a $€ 326.100$ nel gruppo di trattamento con atorvastatina e a $€ 20.419$ nel gruppo placebo. Analizzando la spesa ospedaliera, i costi sono stati maggiori nel gruppo placebo, rispetto al gruppo atorvastatina ( $€ 444.399$ vs $€ 276.086$; riduzione relativa: $-38 \%$ ). Complessivamente, l'aggiunta di atorvastatina alla terapia standard ha generato un aumento dei costi di $€ 137.368$, a fronte però di una riduzione del numero di soggetti colpiti da un evento coronarico ( 32 soggetti liberi da malattia su 1.000 trattati) e di un guadagno complessivo di 29,28 anni di vita (LYG). Nel gruppo di trattamento con atorvastatina $10 \mathrm{mg} / \mathrm{die}$, il costo della terapia costituisce il $54,2 \%$ dei costi totali sanitari (Figura 1). Il rapporto incrementale di costoefficacia (ICER) è pari a $€ 4.299$ per paziente libero da malattia e a $€ 4.692$ per anno di vita guadagnato. I risultati dell'analisi di sensibilità sono mostrati in Tabella III. L'ICER varia da $€ 3.648$ a $€ 5.736$ per LYG al variare del \pm $10 \%$ del costo della terapia, e da $€ 4.117$ a $€$ 5.266 per LYG al variare del $\pm 10 \%$ del costo dei ricoveri. Infine, l'analisi di soglia ha determinato che il trattamento con atorvastatina sarebbe dominante (meno costoso e più efficace rispetto a placebo), se il costo giornaliero fosse inferiore a $€ 0,20$.

\section{CONCLUSIONI}

Nello studio CARDS, atorvastatina $10 \mathrm{mg}$ risulta costo-efficace rispetto a placebo nel ridurre il rischio di evento cardio-cerebrovascolare (ICER: $€ 4.229$ per paziente libero da malattia). Il costo-terapia di atorvastatina $(+306 €$ per paziente) è compensato dalla riduzione della spesa per ricoveri (-168 € per paziente).

\section{DISCUSSIONE}

Il presente studio costituisce un aggiornamento di una precedente analisi economica [10] che ha valutato i costi e i benefici della terapia con atorvastatina in soggetti affetti da diabete mellito di tipo II, con anamnesi negativa di malattie cardiovascolari maggiori. L'aggiornamento è stato reso necessario dalle modifiche dei costi ospedalieri avvenute durante questi ultimi anni, e soprattutto alla luce della riduzione dei costi di atorvastatina a seguito della perdita della prevenzione brevettuale. Lo studio ha dimostrato come atorvastatina possa considerarsi un'alternativa costo-efficace rispetto a placebo nel ridurre l'incidenza di eventi cardiovascolari e nell'aumentare la sopravvivenza complessiva di soggetti affetti da diabete mellito di tipo II. La riduzione del costo di atorvastatina ha ridotto il rapporto incrementale di costo-efficacia a $€ 4.692$ per anno di vita guadagnato (LYG), rispetto a $€ 36.566$ per anno di vita guadagnato calcolato nella precedente analisi [10]. Anche potenziali variazioni dei costi per la terapia farmacologica e per i ricoveri, valutate attraverso l'analisi di sensibilità, hanno confermano la validità dei risultati dell'analisi.

La mancanza di alcune informazioni cliniche riguardanti lo studio CARDS ha imposto diverse ipotesi e approssimazioni che potrebbero influenzare i risultati della presente analisi economica. In ogni caso, comunque, tutte le ipotesi sono state fatte in modo da risultare conservative del possibile vantaggio economico ottenibile con atorvastatina. Per il calcolo dei costi della terapia farmacologica, per esempio, lo studio CARDS ha soltanto riportato la percentuale di soggetti che ogni anno ha ricevuto almeno una dose di farmaco, senza specificare la compliance media dei pazienti. In assenza di informazioni più precise abbiamo ipotizzato una compliance del $100 \%$, che quasi sicuramente sovrastima i costi di trattamento. Per quanto riguarda il costo di gestione dei ricoveri ospedalieri, la nostra analisi non ha permesso di prendere in considerazione gli eventi car-

\section{Figura 1}

Studio CARDS:

ripartizione dei costi sanitari diretti (per 1.000 pazienti, per 3,9 anni; i costi sostenuti negli anni successivi al primo sono scontati del 3\%)

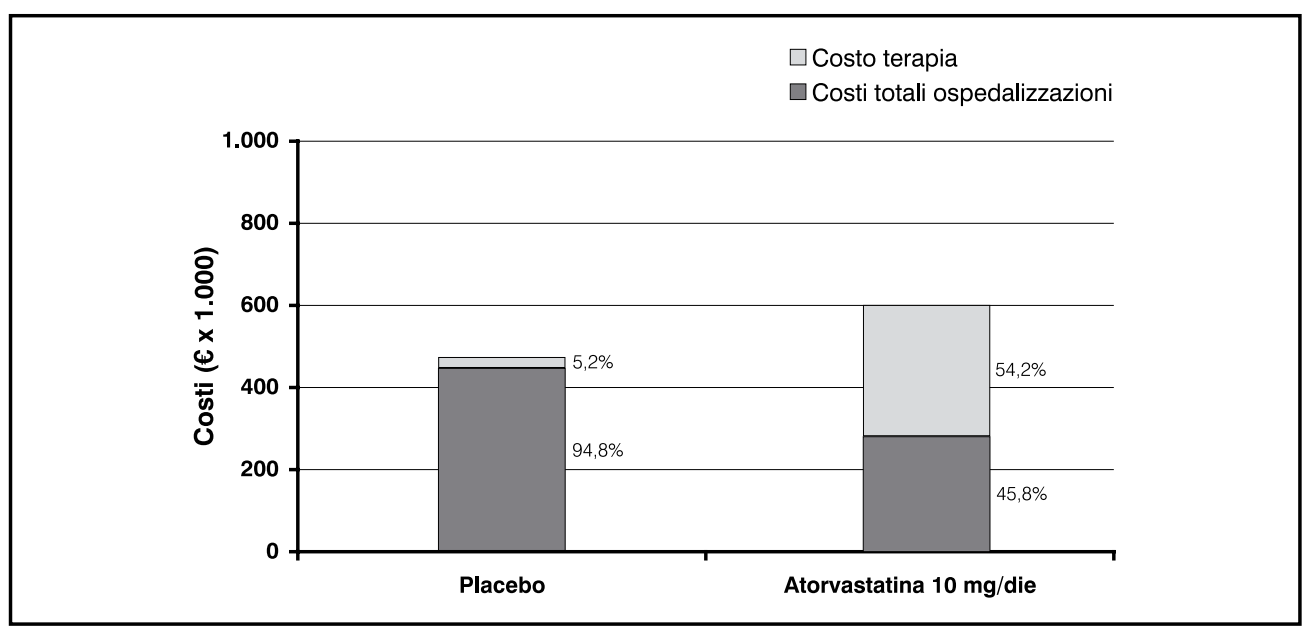


diovascolari minori, la cui entità non è immediatamente deducibile dai dati in possesso. Anche in questo caso, comunque, la frequenza complessiva di eventi cardiovascolari è risultata significativamente superiore nel gruppo di trattamento con placebo $(13,4 \%)$ rispetto ad atorvastatina $(9,4 \% ; p=0,001)$, il che conduce alla conclusione che il risparmio economico per riduzione degli eventi attribuibile alla terapia con atorvastatina possa essere superiore rispetto alla nostra stima. L'analisi non ha infine stimato i costi di gestione della fase post-acuta degli eventi, che incidono significativamente sulla spesa sanitaria complessiva $[12,17]$. In una recente analisi sui costi di gestione di pazien-

\begin{tabular}{lcc}
\hline \multicolumn{1}{c}{ Descrizione } & $\begin{array}{c}\text { Differenza costi }(\boldsymbol{\epsilon}) \\
\text { (Atorvastatina - Placebo) }\end{array}$ & $\begin{array}{c}\text { ICER } \\
(\mathbf{E} / \text { LYG) }\end{array}$ \\
\hline $\pm 10 \%$ costi trattamento & $167.937 ; 106.800$ & $5.736 ; 3.648$ \\
$\pm 10 \%$ costi ospedalizzazioni & $120.537 ; 154.200$ & $4.117 ; 5.266$ \\
\hline
\end{tabular}

\section{Tabella III}

Studio CARDS: analisi di sensibilità

* dati riferiti a una ipotetica coorte di 1.000 soggetti

ti colpiti da infarto del miocardio [12] è stato dimostrato che i costi diretti sanitari sostenuti nell'anno successivo ad un infarto sono superiori rispetto al costo del ricovero stesso.

\section{BIBLIOGRAFIA}

1. International Diabetes Federation. The Diabetes Atlas. Third Edition. Brussels: International Diabetes Federation, 2006

2. Wild S, Roglic G, Green A, Sicree R, King H. Global prevalence of diabetes: estimates for the year 2000 and projections for 2030. Diabetes Care 2004; 27: 1047-53

3. Garancini MP. L'epidemiologia del diabete non-insulino dipendente e della ridotta tolleranza al glucosio. In: Società Italiana di Diabetologia. Il diabete in Italia. Milano: Editrice Kurtis, 1996

4. Jönsson B; CODE-2 Advisory Board. Revealing the cost of Type II diabetes in Europe. Diabetologia 2002; 45: S5-S12

5. Zaniolo O. Costi del diabete in Italia. Farmacoeconomia e percorsi terapeutici 2009; 10: 73-81

6. Rydén L, Standl E, Bartnik M, Van den Berghe G, Betteridge J, de Boer MJ et al.; Task Force on Diabetes and Cardiovascular Diseases of the European Society of Cardiology (ESC); European Association for the Study of Diabetes (EASD). Guidelines on diabetes, pre-diabetes, and cardiovascular diseases: full text. Eur Heart J 2007 : 9(suppl C): C3-C74

7. AMD, FAND, FIC, Forum per la Prevenzione delle Malattie Cardiovascolari, Gruppo Cochrane Collaboration Italia, SID, SIIA, SIMG, SISA. Linee Guida per La Prevenzione Cardiovascolare nel Paziente Diabetico. Ital heart J 2002; 3: 669-76

8. Sever PS, Dahlof B, Poulter NR, Wedel H, Beevers G, Caulfield M et al.; ASCOT investigators. Prevention of coronary and stroke events with atorvastatin in hypertensive patients who have average or lower-than-average cholesterol concentrations, in the Anglo-Scandinavian Cardiac Outcomes Trial-Lipid Lowering Arm (ASCOT-LLA): a multicentre randomized controlled trial. Lancet 2003; 361: 1149-58

9. Colhoun HM, Betteridge DJ, Durrington PN, Hitman GA, Neil HA, Livingstone SJ et al.; CARDS investigators. Primary prevention of cardiovascular disease with atorvastatin in type 2 diabetes in the Collaborative Atorvastatin Diabetes Study (CARDS): multicentre randomized placebo controlled trial. Lancet 2004; 364: 685-96

10. Da Portu S, Menditto E, Mantovani LG, Valutazione economica dello studio CARDS Farmeconomia e percorsi terapeutici 2006; 7: 193-7

11. Codifa ${ }^{\odot}$ : banca dati del farmaco. Ultima consultazione gennaio 2011

12. Mantovani LG, Fornari C, Madotto F, Riva M, Chiodini V, Ferrario MM et al. Cost and Outcomes after First Acute Myocardial Infarction: Study on 12.049 Individuals Using Administrative Databases. ISPOR 2009, Orlando

13. Weinstein Mc, Stason WB. On the foundations of cost-effectiveness analysis for health and medical practices. $N$ Engl J Med 1977; 296: 716-21

14. Drummond MF, Stoddart GL, Torrance GW. Methods for economic evaluation of Health Care Programmes. Oxford: Oxford University Press, 1997

15. Briggs A, Sculpher M, Buxton M. Uncertainty in the economic evaluation of health care technologies: the role of sensitivity analysis. Health Econ 1994; 3: 95-104 
Valutazione economica dello studio CARDS: un aggiornamento

16. Garattini L, Grilli R, Scopelliti D, Mantovani L. A proposal for Italian guidelines in pharmacoeconomics. Pharmacoeconomics 1995; 7: 1-6

17. Morsanutto A, Mantovani LG, Ros B, De Portu S, Spazzapan D, Tosolini F. Costs And Outcomes after First Stroke Hospital Admission: A Longitudinal Study Using Administrative Databases. Value in Health 2005; 8 